\title{
Criteria for selecting microhaplotypes: mixture detection and deconvolution
}

\author{
Kenneth K Kidd ${ }^{*}$ and William C Speed
}

\begin{abstract}
Background: DNA sequencing is likely to become a standard typing method in forensics in the near future. We define a microhaplotype to be a locus with two or more single nucleotide polymorphisms (SNPS) that occur within a short segment of DNA (e.g., 200 bp) that can be covered by a single sequence run and collectively define a multiallelic locus. Microhaplotypes can be highly informative for many forensic questions, including detection of mixtures of two or more sources in a DNA sample, a common problem in forensic practice.

Results: When all alleles are equally frequent, the probability of detecting three or more alleles in a mixture is at maximum. The classical population genetics concept of effective number of alleles at a locus, termed $A_{e}$, converts the unequal allele frequencies at a locus into a value that is equivalent to some number of equally frequent alleles, allowing microhaplotype loci to be ranked. The expectations for the ability to qualitatively detect mixtures are given for different integer values of $A_{e}$ and the cumulative probabilities of detecting mixtures based on testing multiple microhaps are shown to exceed $95 \%$ with as few as five loci with average $A_{e}$ values of even slightly greater than 3.0.
\end{abstract}

Conclusions: Microhaplotypes with $A_{e}$ values of $>3$ will be exceedingly useful in ordinary forensic practice. Based on our studies, 3-SNP microhaplotypes will sometimes meet this criterion, but 4-SNP microhaplotypes can even exceed this criterion and have values $>4$.

Keywords: Microhaplotype, DNA mixtures, Forensic identification, Population genetics

\section{Background}

With the arrival of inexpensive DNA sequencing appropriate for forensic applications, we have reexamined the optimal type of genetic marker for forensic applications. It is clear that several relevant forensic questions cannot be answered by the existing or expanded set of short tandem repeat polymorphisms (STRPs) used by combined DNA index system (CODIS) [1,2]. The CODIS loci or their equivalent STRPs in other countries are good for individual identification, their original and still primary use. While most CODIS loci can be adequately genotyped by sequencing, massively parallel sequencing (MPS) methodology allows other types of markers to be included in the genotyping.

Single nucleotide polymorphisms (SNPs) can enhance the individual identification statistics beyond what the STRPs provide either as nucleotide variants within individual

\footnotetext{
* Correspondence: Kenneth.Kidd@yale.edu

Department of Genetics, Yale University School of Medicine, 333 Cedar Street, PO Box 208005, New Haven, CT 06520-8005, USA
}

STRP alleles or as a separate set of markers [3-5]. By their multiallelic nature STRPs both provide familial relationship information and also can be used to identify and resolve mixtures of DNA from two or more individuals in a single sample. Unfortunately, individual di-allelic SNPs can provide only weak evidence of familial relationships or resolution of mixtures precisely because there are only two alleles. However, sets of SNPs and/or small insertion-deletion polymorphisms (DIPs or Indels) can provide robust information on biogeographic ancestry [6-10], a type of investigative information that panels of STRPs, such as the CODIS loci, cannot provide due to their high global heterozygosity and greater mutation rate. Some SNP genotypes are highly correlated with physical phenotypic traits such as pigmentation of skin/hair/eye [11-13], another form of information that STRPs cannot provide.

Our lab has long used minihaplotypes (minihaps) in anthropological studies [14-16]. These genomic regions of $10 \mathrm{~kb}$ or longer with multiple SNPs of high heterozygosity 
and minimal intermarker recombination can have great value for ancestry determination and anthropology, since the statistical inference of phase can be very accurate. The two areas of forensic analysis that individual SNPs and minihaps cannot address well, familial/lineage information and mixture detection and deconvolution, can be addressed by a new type of marker, SNPs grouped into microhaplotypes (microhaps). Microhaps are defined as loci of two or more SNPs within the span of a single sequence run (arbitrarily set currently at $200 \mathrm{bp}$ ) with three or more common allelic combinations (haplotypes) of the SNPs $[17,18]$. Microhaps properly selected to also avoid recombination hot spots will have mutation rates much lower than those of the STRPs.

In our initial studies $[17,18]$ of many multiallelic microhaps, we have shown that when genotyped by MPS, microhaps can fulfill all the forensic objectives for which the STRPs were originally selected and are now used. Use of MPS obviates the problems of multiplex-ability, sensitivity, and assay cost associated with individual SNP genotyping. In addition to being useful for identification and lineage/family relationships, microhaps can provide information on biogeographic ancestry and can be useful for both detecting and deconvoluting mixtures of DNA. The issue now is identifying and fully characterizing a set of microhaps with the optimum characteristics for specific purposes. We are screening existing databases for appropriate candidate regions and then testing our own panel of 54 population samples to confirm and expand the data. Only microhaps with three or more alleles are of value, and many close SNPs have complete linkage disequilibrium (LD) with only two extant haplotypes (alleles) (cf. discussion in [18]). Given the potentially high heterozygosity of microhaps with three or more alleles, the genotypic uniqueness of individuals for identification is not a major issue: random match probabilities can easily be below $10^{-30}$ with fewer than 50 loci [18]. However, the optimal characteristics of loci differ for the other purposes, as we have explained in developing our panels of single SNPs for identification $[4,19]$ and ancestry [5]. For ancestry inference, the allele frequencies of the loci used must show variation among the populations being considered. For lineage studies, the loci should be highly heterozygous with multiple alleles; yet, that criterion alone may not be sufficient for optimal detection of mixtures.

\section{Methods}

\section{Developing criteria for selecting loci to detect mixtures}

Heterozygosity is the maximum possible when all alleles are equally frequent, and this clearly is optimal for individual identification for any specified number of alleles. For ancestry inference, high heterozygosity is less important than frequency variation among populations. However, in using large datasets generated from either chip-based genotyping or from whole-genome MPS, we have found that many of the loci that have the highest variation among populations represent either genotyping error or assembly errors in MPS analysis for the population(s) with more deviant frequencies. For lineage and ancestry inference, identity by descent (IBD) is important. Loci that may be hypervariable due to frequent mutation and/or recombination would complicate determining IBD among extended relatives or within a tribe of moderate size. Therefore, we have chosen to focus on loci that have most alleles at $>5 \%$ (arbitrarily chosen); a frequency greater than frequent recombination is likely to generate and a frequency greater than typing errors. We could also limit selection to loci that have only the $n+1$ alleles that can be generated by $n$ accumulated mutations. However, we often see an allele (haplotype) that could only have arisen by a crossover among the SNPs within a microhap. In many cases, those appear to be single historical crossover products that have drifted to high frequency and are not recurring at a meaningful frequency of, say, $>10^{-4}$. These considerations help us avoid pursuit of loci that may be hypervariable due to frequent mutation, frequent recombination, and/or errors in the datasets screened.

Given the above consideration, how do we maximize the ability to detect mixtures? We are interested in qualitative determination that a mixture is present in a forensic sample. That occurs when three or more distinct alleles are observed. Obviously, an infinite number of alleles maximizes uniqueness of individuals and hence the difference between any two individuals. To consider the issue for a small finite number of alleles, we have started with the simplest case of three alleles. To evaluate the probability of a mixture having more than two alleles present, we have simply used a multinomial expansion to calculate the probability of a mixture of DNA from two independent individuals having at least three different alleles as a function of the allele frequencies. The function for three alleles is simply $(p+q+r)^{4}=(p+$ $q+r)^{2}(p+q+r)^{2}$, which is the product of the HardyWeinberg genotype arrays for two random unrelated individuals. As shown in Figure 1, the sum of the terms in which three alleles occur is maximized when the alleles are equally frequent. This is also the situation that maximizes heterozygosity. When there are four alleles, $(p+q+r+s)^{4}$, the maximum occurs when all alleles are equally frequent. Conceptually, this maximum occurs at the center of a tetrahedron with each face identical to Figure 1. The overall pattern generalizes to any number of alleles. Table 1 gives those maximum values for loci with 3,4 , and 5 equally frequent alleles. The maximum probability of three or more alleles in a mixture occurs when all alleles at a locus are equally frequent. Obviously, with multiple loci, the maximum 


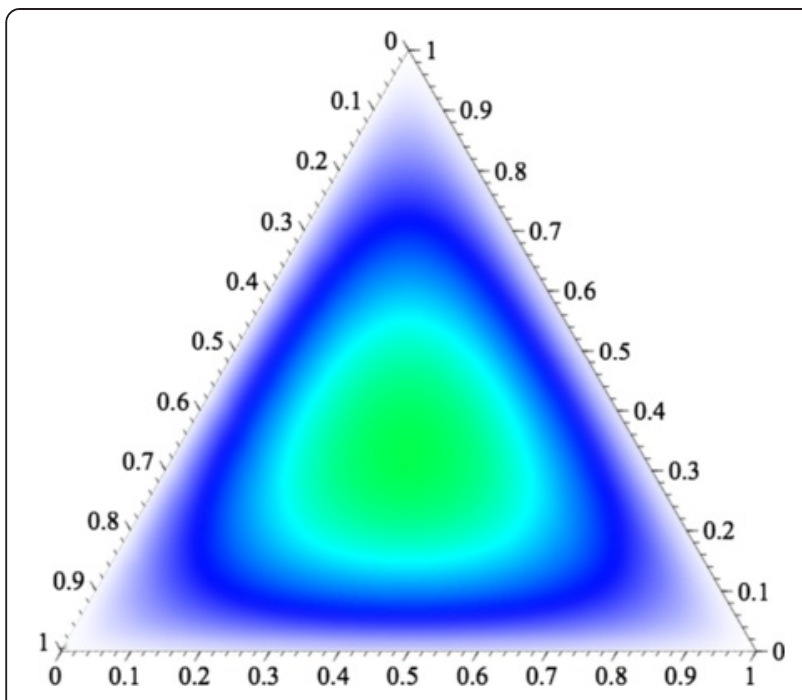

Figure 1 Ternary plot of the probability of a qualitatively detectable mixture. The probability of having more than two haplotypes present, for a three locus system with allele frequencies of $p, q$, and $r$, is calculated for a set of genotypes from a random pair of individuals. The values range from zero along the margins with only two alleles present to the maximum at the "center" where all alleles are equally frequent.

probability will increase as a function of the values at each individual locus.

In searching the HGDP [20,21] or 1000 Genomes [22] databases for optimal microhaps, we have often found either relatively uninformative loci with only one or two really common alleles or many alleles in very unequal frequencies. The loci with only two alleles are easily discarded but the question remains of how to rank the loci for mixture detection when there are multiple alleles at very different frequencies. Classical population genetics gives the answer with the concept of effective number of alleles [23] which we symbolize as $A_{e} . A_{e}$ is defined for a locus as the equivalent number of equally frequent neutral alleles in terms of population dynamics. It is calculated as the reciprocal of

\begin{tabular}{|c|c|c|c|}
\hline $\begin{array}{l}\text { Number of } \\
\text { equally frequent } \\
\text { alleles }\end{array}$ & $\begin{array}{l}\text { Probability of } \\
\text { three alleles } \\
\text { being present }\end{array}$ & $\begin{array}{l}\text { Probability } \\
\text { of four alleles } \\
\text { being present }\end{array}$ & $\begin{array}{l}\text { Total probability } \\
\text { of detecting more } \\
\text { than two alleles } \\
\text { in a mixture }\end{array}$ \\
\hline Three & 0.4444 & - & 0.4444 \\
\hline Four & 0.5625 & 0.09375 & 0.65625 \\
\hline Five & 0.5760 & 0.1920 & 0.7680 \\
\hline
\end{tabular}

Maximum probabilities of detecting a mixture of two random unrelated individuals for three-, four-, and five-allele microhaps. These are the values when all alleles are equally frequent. As shown in Figure 1, the values are lower when the frequencies are not equal. the homozygosity: ${ }^{1} / \sum p_{i}^{2}$ where $p_{i}$ equals the frequency of allele $i$ and summation is over all alleles at the locus.

We know that the potential to detect a mixture increases with more equally frequent alleles (Table 1). Using effective number of alleles converts each locus to the same "standard" and the higher the effective number of alleles, the more probable a mixture could be detected. This applies to a single population but one must also consider variation among populations since the forensic caseload potentially involves individuals from populations originating from many different parts of the world. Therefore, we are using our large set of populations to confirm the variation seen in the initial screenings as well as to extend knowledge to many additional populations.

The $\mathrm{A}_{\mathrm{e}}$ calculation allows the ranking of loci by their probabilities of qualitatively detecting a mixture. In reality, several loci will be used in a forensic analysis. If we select loci with an average $\mathrm{A}_{\mathrm{e}}$ of 4 , then we can calculate the probability of "detecting" a mixture with at least one of $n$ loci as $1-(1-.65625)^{n}$. Table 2 gives the probability of "detecting" a mixture qualitatively for different numbers of loci studied at integral values of $A_{e}$ from 3 to 5 . Obviously, mixtures of loci with different $A_{e}$ values will give intermediate results as will loci with non-integer $A_{e}$ values between 3 and 5 . We have not extended the table beyond 5 loci since the probability rapidly approaches certainty and we have yet to find a locus with a global average $A_{e}$ of 5 or greater. Nor have we considered numerically the issue of a mixture of more than two individuals, but the logic applies to such cases as well and detecting five alleles indicates at least a three person mixture.

\section{Results and discussion}

After identifying a potentially useful microhap in large public data sets, we used our set of DNA samples from over 2,500 individuals originating from 54 populations to confirm the pattern of variation and obtain statistics from a global sample of populations [5]. Our initial studies involved microhaps with only two or three SNPs $[17,18]$. We started with those because they were the simplest to find, often involving SNPs we had already tested on the 54 populations we are routinely studying.

Table 2 Cumulative probability of a mixture having three or more alleles at two or more loci

\begin{tabular}{lllll}
\hline \multirow{2}{*}{ Effective number of alleles, $\mathbf{A}_{\mathbf{e}}$} & \multicolumn{4}{l}{ Number of loci studied } \\
\cline { 2 - 5 } & $\mathbf{2}$ & $\mathbf{3}$ & $\mathbf{4}$ & $\mathbf{5}$ \\
\hline 3 & 0.69131 & 0.82849 & 0.90471 & 0.94706 \\
4 & 0.88184 & 0.95938 & 0.98604 & 0.9952 \\
5 & 0.94618 & 0.98751 & 0.99710 & 0.99933 \\
\hline
\end{tabular}

Cumulative probability of a mixture having three or more alleles at two or more loci, for integral values of $A_{e}$. See text. 
The global average $A_{e}$ for the 31 microhaps published [18] ranges from 1.9 to 2.8 (Figure 2). When we recognized that the maximum power to detect mixtures is a function of $\mathrm{A}_{e}$, we decided to use $\mathrm{A}_{\mathrm{e}}$ as an effective way to compare loci and to focus on microhaps with an $A_{e}>$ 3.0. Microhaps with four SNPs are an interesting subset to consider because they can have an $\mathrm{A}_{\mathrm{e}}$ considerably greater than 3.0. While there are potential microhaps with more than four SNPs, we found that the pool of potential microhaps with SNP frequency data for all SNPs in the microhap, and with individual SNP heterozygosities above $18 \%$, was very small. By focusing on 4SNP microhaps, there still remained a large enough pool to be able to look at frequency distributions and the pairwise correlations between SNP frequencies in the microhaplotype. We developed empiric thresholds based on these distributions. In our search of the $1000 \mathrm{Ge}$ nomes data using criteria described in Additional file 1, we identified 341 microhaps comprised of four SNPs. We calculated $A_{e}$ for each locus in each population using the haplotype frequencies calculated by PHASE [24]. Considering the matrix of 341 microhaps $\times 20$ populations, the row and column averages show markedly different ranges. The average $A_{e}$ for each of the 341 microhaps ranged from 1.79 to 6.96 . The average $A_{e}$ for each of the 20 populations ranged from 2.92 to 3.64 .

We are in the process of testing many of these and other microhaps and present here examples of the highest global average $A_{e}$ values among the 2-SNP, the 3-SNP, and the 4-SNP microhaps that we have now tested on over 60 populations (Table 3). The examples range from an $\mathrm{A}_{\mathrm{e}}$ of 2.7 for the second highest 2-SNP microhap to just over 4.7 for the highest 4-SNP microhap.

Figures 3, 4, and 5 show population-specific allele frequencies for the six microhaps in Table 3 for a global set of populations. The allele frequencies clearly vary among populations, some of which reflects sampling error, but clear differences also exist among biogeographic regions for some of these loci. We can rank the loci by the $A_{e}$ for a specific population of interest or, since $a$ priori the relevant population(s) may be unknown for a forensic analysis, by the global average $\mathrm{A}_{\mathrm{e}}$. However, it is important to recognize that by selecting for high average $A_{e}$ to maximize mixture detection, we are tending to reduce large regional differences in allele frequencies and thus choose less ancestry informative loci. In future papers, we will discuss criteria for identifying and evaluating optimal microhaps for ancestry and lineage inference.

With two SNPs and a maximum of four haplotypes, we have yet to find a microhap with a global average $A_{e}$ of $>3.0$. While 17 of the 27 two-SNP microhaps we have already published [18] have individual populations with $A_{e}>3.0$, global averages are generally decreased especially due to Native American populations, where decreased genetic diversity means only 4 of the 2-SNP microhaps have $A_{e}>3.0$ in these populations. Microhap048 has all four possible haplotypes but the unequal frequencies result in a global average $A_{e}$ of only 2.71 , the second best of those we have examined. Microhap046 also has all four possible haplotypes and the highest $\mathrm{A}_{\mathrm{e}}$ for the 2-SNP microhaps; the allele frequencies are seemingly more uneven but the global average $A_{e}$ is

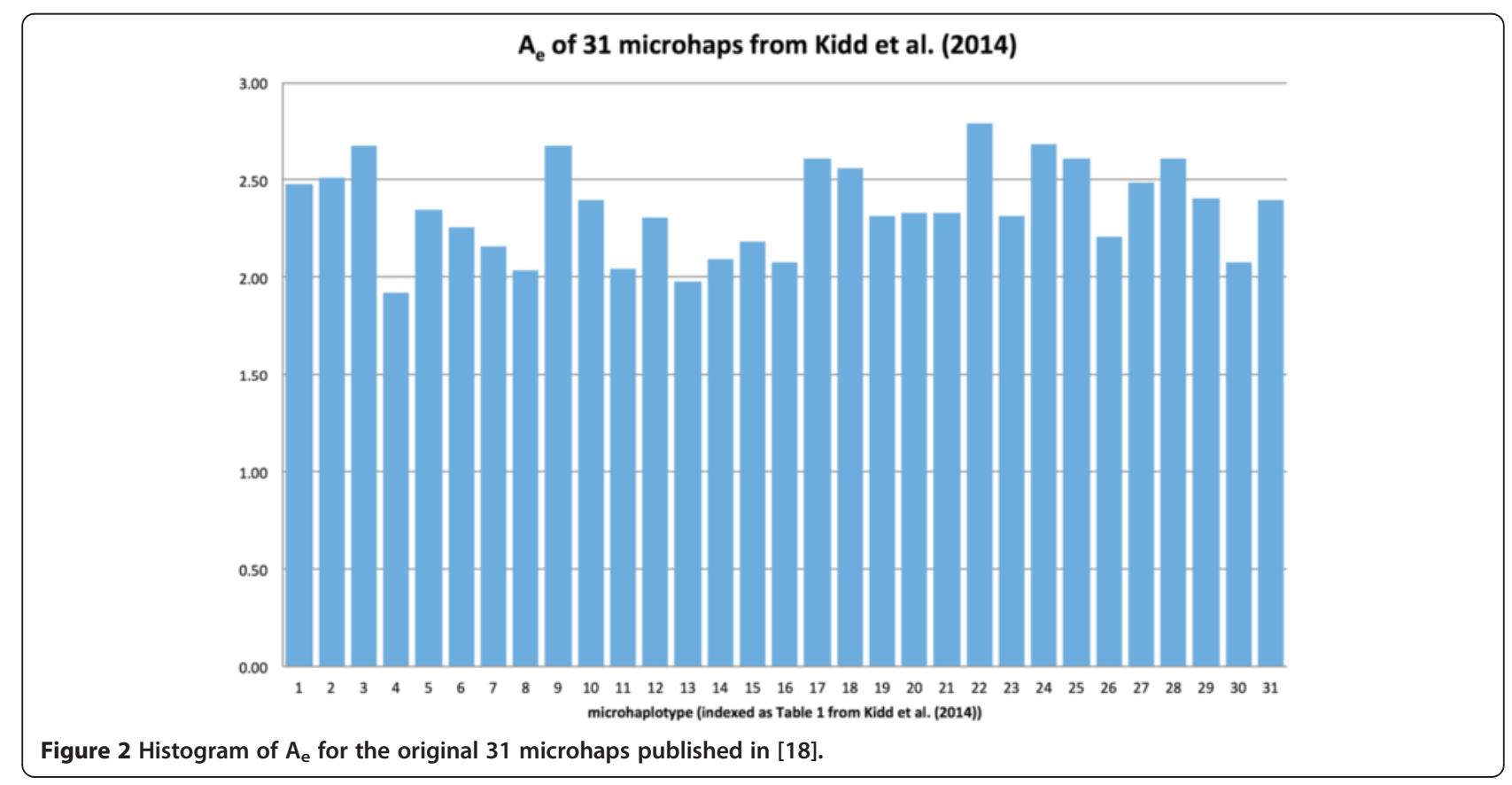


Table 3 Examples of 2-SNP, 3-SNP, and 4-SNP microhaplotypes with largest $A_{e}$ values

\begin{tabular}{|c|c|c|c|}
\hline Provisional locus name & $\begin{array}{l}\text { SNPs } \\
\text { involved }\end{array}$ & $\begin{array}{l}\text { Extent } \\
\text { in bp }\end{array}$ & $\begin{array}{l}\text { Average } \\
\text { global } A_{e}\end{array}$ \\
\hline \multirow[t]{2}{*}{ Microhap048 (mh24:C14ORF43 [18]) } & rs12717560 & 159 & 2.708 \\
\hline & rs12878166 & & \\
\hline \multirow[t]{2}{*}{ Microhap046 (mh22:SUDS3 [18]) } & rs1503767 & 72 & 2.842 \\
\hline & rs11068953 & & \\
\hline \multirow[t]{3}{*}{ Microhap049 } & rs9937467 & 59 & 2.888 \\
\hline & rs17670098 & & \\
\hline & rs17670111 & & \\
\hline \multirow[t]{3}{*}{ MicroHap061 } & rs763040 & 146 & 3.192 \\
\hline & rs5764924 & & \\
\hline & rs763041 & & \\
\hline \multirow[t]{4}{*}{ MicroTetrad180 } & rs12802112 & 193 & 4.008 \\
\hline & rs28631755 & & \\
\hline & rs7112918 & & \\
\hline & rs4752777 & & \\
\hline \multirow[t]{4}{*}{ MicroTetrad315 } & rs8126597 & 145 & 4.763 \\
\hline & rs6517970 & & \\
\hline & rs8131148 & & \\
\hline & rs6517971 & & \\
\hline
\end{tabular}

Examples of 2-SNP, 3-SNP, and 4-SNP microhaplotypes with largest $A_{e}$ values characterized on our laboratory's populations to date. The 2-SNP microhaps were published in [18] under the locus name appended to the "Provisional Locus Name" field; The microhap number indicates the number of that locus in [18] and in Figure 2.

slightly larger, 2.84. The 3-SNP microhaps allow for a maximum of eight microhaps, but our selection criteria have generally identified loci with fewer than eight alleles. Microhap049 is the second best with five of the eight possible haplotypes at common frequencies in all regions of the world with an additional microhap seen only in the Americas. The global average $\mathrm{A}_{\mathrm{e}}$ of 2.89 is barely larger than the best of the 2-SNP microhaps. Microhap061 also has five haplotypes with similar frequencies among most populations except Native Americans and has a global average $A_{e}$ of 3.12. The 4-SNP microhaps we have studied so far allow a much greater number of alleles and the two illustrated show considerable within-population variation. Microhap Tetrad180 has six haplotypes that occur essentially globally, and its $\mathrm{A}_{\mathrm{e}}$ of 4.008 is the second best. Finally, microhap Tetrad315 has the most allelic complexity with 10 of the 16 possible haplotypes reaching frequencies $>5 \%$ in many populations and a global average $A_{e}$ of 4.76 , the highest global average we have found to date.

Microhap Tetrad315 illustrates our concern about microhaps with frequent recombination for ancestry inference. We have not yet been able to document that most of the variation is old and not frequently regenerated although it is clear that certain combinations among the more common haplotypes are not seen at the frequencies that might occur if recombination were very frequent. Even a high recombination rate of $1 \%$ would still allow meaningful lineage inference with several loci such as this. However, that concern is not relevant to individual identification and mixture detection and deconvolution. Those rely on the allele/haplotype frequencies in the population, not on the ongoing origins of the alleles or identity by recent descent of the different alleles. This locus and the other 4-SNP microhap illustrated, Tetrad180, will be able to provide excellent mixture detection ability.

The calculations we have made assume that the contributions from the two individuals can be detected. Issues of sensitivity enter when the contributions are not equal, as is almost always the case in forensics. Those issues are dependent on the method used and, since we assume sequencing will be used, on the depth of coverage, i.e., the number of reads per locus. With an average of $>100$ reads per allele, a minimal expectation for MPS, a third allele can be said to be present if several reads detect it. With experience, not yet available, the community can decide on the criteria necessary to overcome the stochastic aspect of number of reads of an allele. With a clear stochastic threshold, quantitative estimation of the relative contributions of individuals to a mixture is clearly possible. We note that these concerns are no different from those currently used for the CODIS STRPs. Considerable experience exists with these STRPs yet there are different criteria used in different labs to evaluate whether a low RFU peak represents chance or a real peak. With MPS, even very low levels of a second contributor to a mixture could result in multiple reads with a unique combination of SNP alleles (a unique haplotype) on single reads, giving multiple confirmation of the presence of more than one DNA. In contrast to many evaluations of mixtures using STRPs that require decisions surrounding stutter peaks and low RFU peaks in mixtures, MPS microhaps are clearly superior. We think that sequence will have less ambiguity at specific nucleotides and make low levels of mixture detectable, but actual experience is needed.

To date, we have evaluated candidate microhaps using TaqMan assays to detect the individual SNPs that we know from our database screens will very likely yield useful loci. If we had sequencing data instead of genotype data, we would be able to show greater $A_{e}$ measures due to cryptic variation unseen by our pilot SNP-typing method. A rare SNP documented at $1 \%$ most likely subdivides some haplotype. Similarly, even the one-off variants already documented will provide what is likely a rare unique haplotype. From the available data, one cannot estimate how the combinations of these untested 


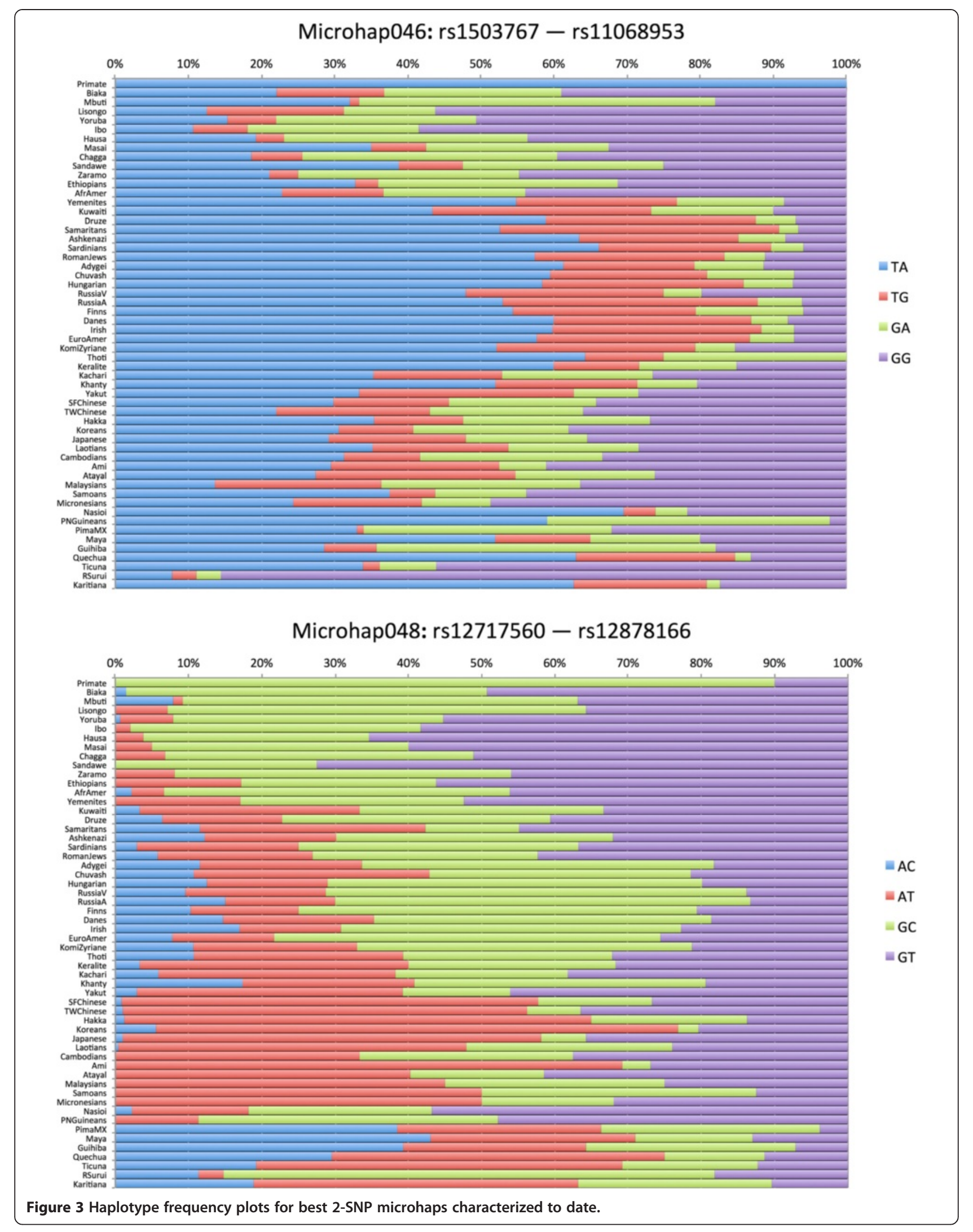




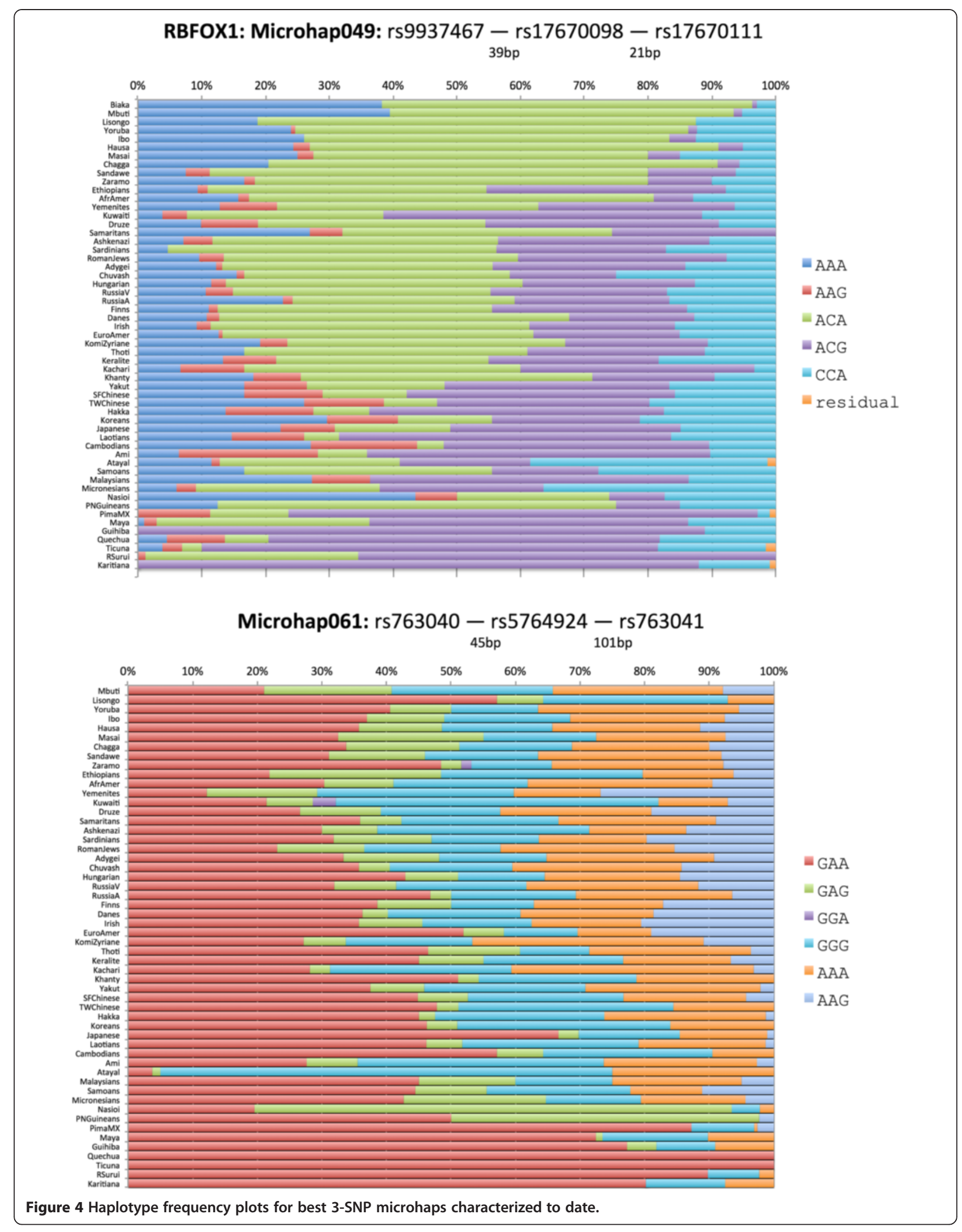




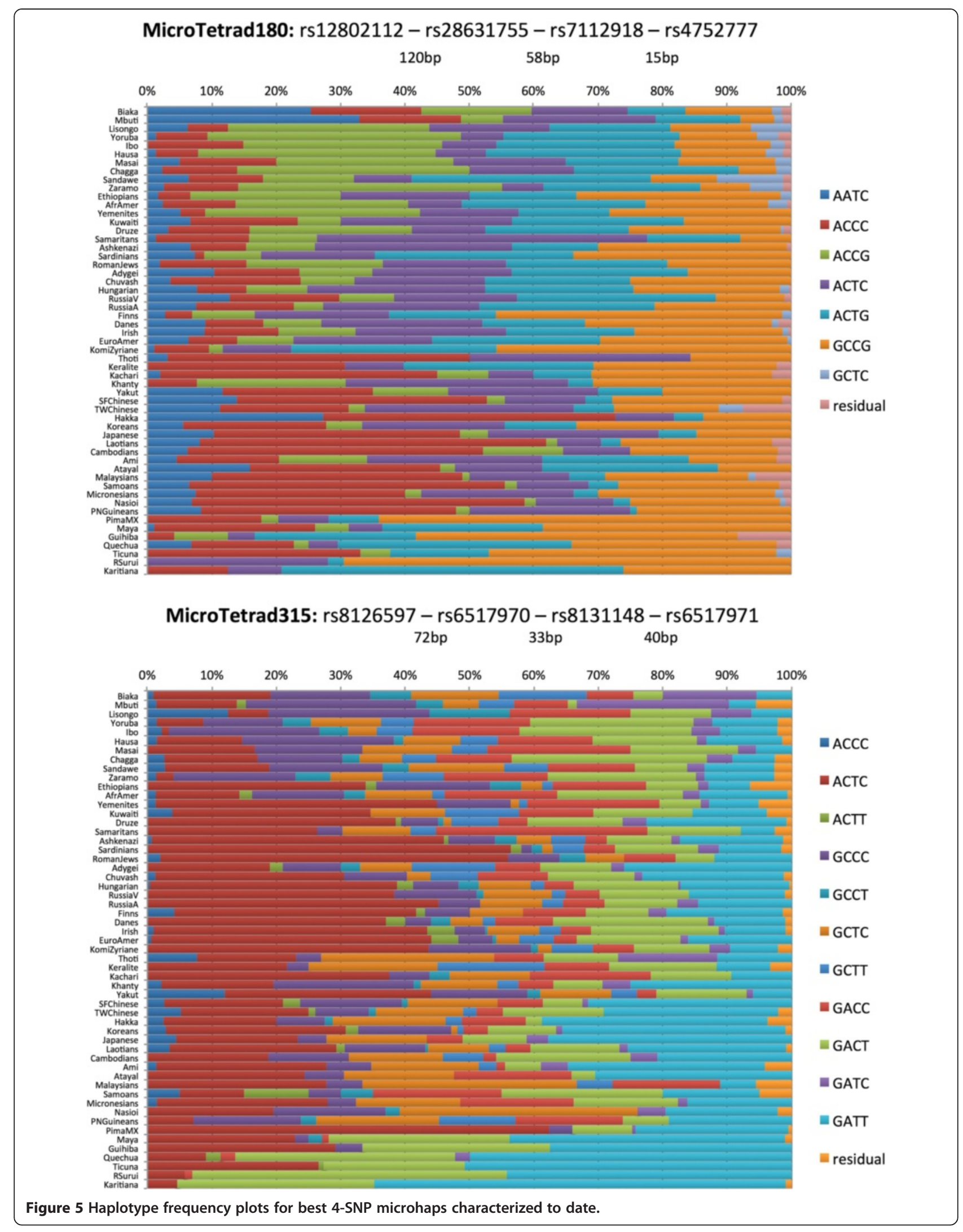




\section{Authors' contributions}

Both authors contributed equally in all aspects of the study including writing of the paper. Both authors read and approved the manuscript.

\section{Acknowledgements}

The microhaps used as illustrations of $A_{e}$ were selected from a larger ongoing collaborative project on microhaplotypes with Robert Lagacé, Joseph Chang, and Sharon Wootton at Lifetech and Andrew Pakstis in the Kidd Lab.

We thank Eva Haigh for excellent technical work in typing the markers, Françoise Friedlaender for help with some of the figures, and Andrew Pakstis and Judith Kidd for comments on the manuscript.

We thank the thousands of individuals who volunteered to give the DNA samples that allowed this study to be done and the many collaborators who helped collect the samples over the past 29 years. In addition, some of the cell lines were obtained from the National Laboratory for the Genetics of Israeli Populations at Tel Aviv University, and the African American samples were obtained from the Coriell Institute for Medical Research, Camden, New Jersey.

This study was supported in part by NIJ Grants 2010-DN-BX-K225 and 2013-DNBX-K023 to KKK awarded by the National Institute of Justice, Office of Justice Programs, U.S. Department of Justice. Points of view in this presentation are those of the authors and do not necessarily represent the official position or policies of the U.S. Department of Justice.

Received: 12 September 2014 Accepted: 10 December 2014

Published online: 28 January 2015

\section{References}

1. Budowle B, Moretti TR, Niezgoda SJ, Brown BL. CODIS and PCR-based short tandem repeat loci: Law Enforcement Tools. In: Proceedings of the Second European Symposium on Human Identification. Madison, Wl: Promega Corporation; 1998. p. 73-88. Available at [http://www.promega.com/ /media/ files/resources/conference\%20proceedings/ishi\%2002/oral\%20 presentations/17.pdf]

2. Federal Bureau of Investigation: FBI-Combined DNA Index System [http://www.fbi.gov/about-us/lab/biometric-analysis/codis]

3. Sanchez JJ, Phillips C, Børsting C, Balogh K, Bogus M, Fondevila M, et al. A multiplex assay with 52 single nucleotide polymorphisms for human identification. Electrophoresis. 2006;27(9):1713-24.

4. Pakstis AJ, Speed WC, Fang R, Hyland FC, Furtado MR, Kidd JR, et al. SNPs for a universal individual identification panel. Hum Genet. 2010;127(3):315-24.

5. Kidd KK, Speed WC, Pakstis AJ, Furtado MR, Fang R, Madbouly A, et al. Progress toward an efficient panel of SNPs for ancestry inference. Forensic Sci Int Genet. 2014;10:23-32.

6. Zaumsegel D, Rothschild MA, Schneider PM. A 21 marker insertion deletion polymorphism panel to study biogeographic ancestry. Forensic Sci Int Genet. 2013;7(2):305-12.

7. Phillips C, Freire Aradas A, Kriegel AK, Fondevila M, Bulbul O, Santos C, et al. Eurasiaplex: a forensic SNP assay for differentiating European and South Asian ancestries. Forensic Sci Int Genet. 2013;7(3):359-66

8. LaRue BL, Ge J, King JL, Budowle B. A validation study of the Qiagen Investigator DIPplex ${ }^{\oplus}$ kit; an INDEL-based assay for human identification. Int J Legal Med. 2012;126(4):533-40.

9. Pereira R, Phillips C, Pinto N, Santos C, dos Santos SE, Amorim A, et al. Straightforward inference of ancestry and admixture proportions through ancestry-informative insertion deletion multiplexing. PLoS One. 2012;7(1):e29684

10. Fondevila M, Pereira R, Gusmao L, Phillips C, Lareu MV, Carracedo A, et al. Forensic performance of two insertion-deletion marker assays. Int J Legal Med. 2012;126:725-37.

11. Walsh S, Liu F, Wollstein A, Kovatsi L, Ralf A, Kosiniak-Kamysz A, et al. The HrrisPlex system for simultaneous prediction of hair and eye colour from DNA. Forensic Sci Int Genet. 2013;7(1):98-115.

12. Maroñas O, Phillips C, Söchtig J, Gomez-Tato A, Cruz R, Alvarez-Dios J, et al. Development of a forensic skin colour predictive test. Forensic Sci Int Genet. 2014;13C:34-44.

13. Gettings KB, Lai R, Johnson JL, Peck MA, Hart JA, Gordish-Dressman H, et al. A 50-SNP assay for biogeographic ancestry and phenotype prediction in the U.S. population. Forensic Sci Int Genet. 2014;8(1):101-8.
14. Kidd JR, Friedlaender F, Pakstis AJ, Furtado M, Fang R, Wang X, et al. SNPS and haplotypes in Native American populations. Am J Phys Anthropol. 2011;146:495-502.

15. Donnelly MP, Paschou P, Grigorenko E, Gurwitz D, Barta C, Lu R-B, et al. A global view of the OCA2-HERC2 region and pigmentation. Hum Genet. 2012;131:683-96.

16. Pakstis AJ, Fang R, Furtado MR, Kidd JR, Kidd KK. Mini-haplotypes as lineage informative SNPS (LISNPS) and ancestry inference SNPS (AISNPS). Eur J Hum Genet. 2012;20:1148-54.

17. Kidd KK, Pakstis AJ, Speed WC, Lagace R, Chang J, Wootton S, et al. Microhaplotype loci are a powerful new type of forensic marker. Melbourne, Australia: 25th Congress of the International Society for Forensic Genetics; 2013

18. Kidd KK, Pakstis AJ, Speed WC, Lagacé R, Chang J, Wootton S, et al. Current sequencing technology makes microhaplotypes a powerful new type of genetic marker for forensics. Forensic Sci Int Genet. 2014;12:215-24.

19. Kidd KK, Kidd JR, Speed WC, Fang R, Furtado MR, Hyland FC, et al. Expanding data and resources for forensic use of SNPs in individual identification. Forensic Sci Int Genet. 2012;6:646-52.

20. Jakobsson M, Scholz SW, Scheet P, Gibbs JR, VanLiere JM, Fung HC, et al. Genotype, haplotype and copy-number variation in worldwide human populations. Nature. 2008;451:998-1003.

21. Li JZ, Absher DM, Tang H, Southwick AM, Casto AM, Ramachandran S, et al. Worldwide human relationships inferred from genome-wide patterns of variation. Science. 2008;319:1100-4.

22. The 1000 Genomes Project Consortium. A map of human genome variation from population-scale sequencing. Nature. 2010;467:1061-73.

23. Crow JF, Kimura M. An Introduction to Population Genetics Theory. New York: Harper \& Row; 1970.

24. Stephens M, Smith N, Donnelly P. A new statistical method for haplotype reconstruction from population data. Am J Hum Genet. 2001;68:978-89.

25. Roman JK, Reid S, Reid J, Chalfin A, Adams W, Knight C. The DNA Field Experiment: Cost-Effectiveness Analysis of the Use of DNA in the Investigation of High-Volume Crimes. Washington DC: The Urban Institute; 2008 [http:// www.urban.org/UploadedPDF/411697_dna_field_experiment.pdf].

26. National Institute of Justice. The Future of Forensic DNA Testing: Predictions of the Research and Development Working Group. Washington DC: U.S. Department of Justice; 2000. p. 43-5.

\section{Submit your next manuscript to BioMed Central and take full advantage of:}

- Convenient online submission

- Thorough peer review

- No space constraints or color figure charges

- Immediate publication on acceptance

- Inclusion in PubMed, CAS, Scopus and Google Scholar

- Research which is freely available for redistribution 